\title{
BERTRAND CURVES IN THREE DIMENSIONAL LIE GROUPS
}

\author{
O. ZEKI OKUYUCU, İSMAİL GÖK, YUSUF YAYLI, AND NEJAT EKMEKCI \\ Received 07 September, 2014
}

\begin{abstract}
In this paper, we give the definition of harmonic curvature function some special curves such as helix, slant curves, Mannheim curves and Bertrand curves. Then, we recall the characterizations of helices [7], slant curves (see [19]) and Mannheim curves (see [12]) in three dimensional Lie groups using their harmonic curvature function.

Moreover, we define Bertrand curves in a three dimensional Lie group $G$ with a bi-invariant metric and the main result in this paper is given as (Theorem 7): A curve $\alpha: I \subset \mathbb{R} \rightarrow G$ with the Frenet apparatus $\{T, N, B, \kappa, \tau\}$ is a Bertrand curve if and only if
\end{abstract}

$$
\lambda \kappa+\mu \kappa H=1
$$

where $\lambda, \mu$ are constants and $H$ is the harmonic curvature function of the curve $\alpha$.

2010 Mathematics Subject Classification: 53A04; 22E15

Keywords: Bertrand curves, Lie groups.

\section{INTRODUCTION}

The general theory of curves in a Euclidean space (or more generally in a Riemannian manifolds) have been developed a long time ago and we have a deep knowledge of its local geometry as well as its global geometry. In the theory of curves in Euclidean space, one of the important and interesting problem is characterizations of a regular curve. In the solution of the problem, the curvature functions $k_{1}$ (or $\left.\kappa\right)$ and $k_{2}$ (or $\tau$ ) of a regular curve have an effective role. For example: if $k_{1}=0=k_{2}$, then the curve is a geodesic or if $k_{1}=$ constant $\neq 0$ and $k_{2}=0$, then the curve is a circle with radius $\left(1 / k_{1}\right)$, etc. Thus we can determine the shape and size of a regular curve by using its curvatures. Another way in the solution of the problem is the relationship between the Frenet vectors of the curves (see [15]).

For instance Bertrand curves: In the classical diferential geometry of curves, J. Bertrand studied curves in Euclidean 3-space whose principal normals are the principal normals of another curve. In [3], he showed that a necessary and sufficient condition for the existence of such a second curve is that a linear relationship with constant coefficients shall exist between the first and second curvatures of the given original curve. In other word, if we denote first and second curvatures of a given curve by $k_{1}$ and $k_{2}$ respectively, then for $\lambda, \mu \in \mathbb{R}$ we have $\lambda k_{1}+\mu k_{2}=1$. Since 
the time of Bertrand's paper, pairs of curves of this kind have been called Conjugate Bertrand Curves, or more commonly Bertrand Curves (see [15]).

In 1888, $C$. Bioche [4] give a new theorem to obtaining Bertrand curves by using the given two curves $C_{1}$ and $C_{2}$ in Euclidean 3-space. Later, in 1960, J. F. Burke [5] give a theorem related with Bioche's thorem on Bertrand curves.

The following properties of Bertrand curves are well known: If two curves have the same principal normals, (i) corresponding points are a fixed distance apart; (ii) the tangents at corresponding points are at a fixed angle. These well known properties of Bertrand curves in Euclidean 3-space was extended by L. R. Pears in [21] to Riemannian $n$-space and found general results for Bertrand curves. When we applying these general result to Euclidean $n$-space, it is easily find that either $k_{2}$ or $k_{3}$ is zero; in other words, Bertrand curves in , $\mathbb{E}^{n}(n>3)$ are degenerate curves. This result is restated by Matsuda and Yorozu [18]. They proved that there is no special Bertrand curves in $E^{n}(n>3)$ and they define new kind, which is called $(1,3)$-type Bertrand curves in 4-dimensional Euclidean space. Bertrand curves and their characterizations were studied by many authours in Euclidean space as well as in Riemann-Otsuki space, in Minkowski 3- space and Minkowski spacetime (for instance see [1,2, 10, 14, 17, 22, 23].)

The degenarete semi-Riemannian geometry of Lie group is studied by Çöken and Çiftçi [8]. Moreover, they obtanied a naturally reductive homogeneous semiRiemannian space using the Lie group. Then Çiftçi [7] defined general helices in three dimensional Lie groups with a bi-invariant metric and obtained a generalization of Lancret's theorem. Also he gave a relation between the geodesics of the so-called cylinders and general helices. Then, Okuyucu et al. [19] defined slant helices in three dimensional Lie groups with a bi-invariant metric and obtained some characterizations using their harmonic curvature function.

Recently, Izumiya and Takeuchi [13] have introduced the concept of slant helix in Euclidean 3 -space. A slant helix in Euclidean space $\mathbb{E}^{3}$ was defined by the property that its principal normal vector field makes a constant angle with a fixed direction. Also, Izumiya and Takeuchi showed that $\alpha$ is a slant helix if and only if the geodesic curvature of spherical image of principal normal indicatrix $(N)$ of a space curve $\alpha$

$$
\sigma_{N}(s)=\left(\frac{\kappa^{2}}{\left(\kappa^{2}+\tau^{2}\right)^{3 / 2}}\left(\frac{\tau}{\kappa}\right)^{\prime}\right)(s)
$$

is a constant function .

Harmonic curvature functions were defined by Özdamar and Hacisalihoğlu [20]. Recently, many studies have been reported on generalized helices and slant helices using the harmonic curvatures in Euclidean spaces and Minkowski spaces [6,11,16]. Then, Okuyucu et al. [19] defined slant helices in three dimensional Lie groups with a bi-invariant metric and obtained some characterizations using their harmonic curvature function. 
In this paper, first of all, we give the definition of harmonic curvature function some special curves such as helix, slant curves. Then, we recall the characterizations of helices [7], slant curves (see [19]) and Mannheim curves (see [12]) in three dimensional Lie groups using their harmonic curvature function. Moreover, we define Bertrand curves in a three dimensional Lie group $G$ with a bi-invariant metric and then the main result to this paper is given as (Theorem 7): A curve $\alpha: I \subset \mathbb{R} \rightarrow G$ with the Frenet apparatus $\{T, N, B, \kappa, \tau\}$ is a Bertrand curve if and only if

$$
\lambda \kappa+\mu \kappa H=1
$$

where $\lambda, \mu$ are constants and $H$ is the harmonic curvature function of the curve $\alpha$.

Note that three dimensional Lie groups admitting bi-invariant metrics are $S O$ (3), $S U^{2}$ and Abelian Lie groups. So we believe that our characterizations about Bertrand curves will be useful for curves theory in Lie groups.

\section{PRELIMINARIES}

Let $G$ be a Lie group with a bi-invariant metric $\langle$,$\rangle and D$ be the Levi-Civita connection of Lie group $G$. If $\mathfrak{g}$ denotes the Lie algebra of $G$ then we know that $\mathfrak{g}$ is isomorphic to $T_{e} G$ where $e$ is neutral element of $G$. If $\langle$,$\rangle is a bi-invariant metric on$ $G$ then we have

$$
\langle X,[Y, Z]\rangle=\langle[X, Y], Z\rangle
$$

and

$$
D_{X} Y=\frac{1}{2}[X, Y]
$$

for all $X, Y$ and $Z \in \mathfrak{g}$.

Let $\alpha: I \subset \mathbb{R} \rightarrow G$ be an arc-lenghted regular curve and $\left\{X_{1}, X_{2}, \ldots, X_{n}\right\}$ be an orthonormal basis of $\mathfrak{g}$. In this case, we write that any two vector fields $W$ and $Z$ along the curve $\alpha$ as $W=\sum_{i=1}^{n} w_{i} X_{i}$ and $Z=\sum_{i=1}^{n} z_{i} X_{i}$ where $w_{i}: I \rightarrow \mathbb{R}$ and $z_{i}: I \rightarrow \mathbb{R}$ are smooth functions. Also the Lie bracket of two vector fields $W$ and $Z$ is given

$$
[W, Z]=\sum_{i=1}^{n} w_{i} z_{i}\left[X_{i}, X_{j}\right]
$$

and the covariant derivative of $W$ along the curve $\alpha$ with the notation $D_{\alpha^{\prime}} W$ is given as follows

$$
D_{\alpha^{\prime}} W=\dot{W}+\frac{1}{2}[T, W]
$$

where $T=\alpha^{\prime}$ and $\dot{W}=\sum_{i=1}^{n} \dot{w}_{i} X_{i}$ or $\dot{W}=\sum_{i=1}^{n} \frac{d w}{d t} X_{i}$. Note that if $W$ is the left-invariant vector field to the curve $\alpha$ then $\dot{W}=0$ (see for details [9]).

Let $G$ be a three dimensional Lie group and $(T, N, B, \kappa, \tau)$ denote the Frenet apparatus of the curve $\alpha$. Then the Serret-Frenet formulas of the curve $\alpha$ satisfies: 


$$
D_{T} T=\kappa N, \quad D_{T} N=-\kappa T+\tau B, \quad D_{T} B=-\tau N
$$

where $D$ is Levi-Civita connection of Lie group $G$ and $\kappa=\|T\|$.

Definition 1 ([7]). Let $\alpha: I \subset \mathbb{R} \rightarrow G$ be a parametrized curve. Then $\alpha$ is called a general helix if it makes a constant angle with a left-invariant vector field $X$. That is,

$$
\langle T(s), X\rangle=\cos \theta \text { for all } s \in I,
$$

for the left-invariant vector field $X \in g$ is unit length and $\theta$ is a constant angle between $X$ and $T$, which is the tangent vector field of the curve $\alpha$.

Proposition 1 ([7]). Let $\alpha: I \subset \mathbb{R} \rightarrow G$ be a parametrized curve with the Frenet apparatus $(T, N, B, \kappa, \tau)$ then $\tau_{G}$ is defined by

or

$$
\tau_{G}=\frac{1}{2}\langle[T, N], B\rangle
$$

$$
\tau_{G}=\frac{1}{2 \kappa^{2} \tau}\langle\ddot{T},[T, \dot{T}]\rangle+\frac{1}{4 \kappa^{2} \tau}\|[T, \dot{T}]\|^{2} .
$$

Definition 2 ([19]). Let $\alpha: I \subset \mathbb{R} \rightarrow G$ be an arc length parametrized curve. Then $\alpha$ is called a slant helix if its principal normal vector field makes a constant angle with a left-invariant vector field $X$ which is unit length. That is,

$$
\langle N(s), X\rangle=\cos \theta \text { for all } s \in I,
$$

where $\theta \neq \frac{\pi}{2}$ is a constant angle between $X$ and $N$ which is the principal normal vector field of the curve $\alpha$.

Definition 3 ([19]). Let $\alpha: I \subset \mathbb{R} \rightarrow G$ be an arc length parametrized curve with the Frenet apparatus $\{T, N, B, \kappa, \tau\}$. Then the harmonic curvature function of the curve $\alpha$ is defined by

where $\tau_{G}=\frac{1}{2}\langle[T, N], B\rangle$.

$$
H=\frac{\tau-\tau_{G}}{\kappa}
$$

Theorem 1 ([7]). Let $\alpha: I \subset \mathbb{R} \rightarrow G$ be a parametrized curve with the Frenet apparatus $(T, N, B, \kappa, \tau)$. The curve $\alpha$ is a general helix, if and only if

$$
\tau=c \kappa+\tau_{G}
$$

where $c$ is a constant.

Also, the next theorem can be given by using the definition of the harmonic curvature function of the curve $\alpha$.

Theorem 2. Let $\alpha: I \subset \mathbb{R} \rightarrow G$ be a parametrized curve with the Frenet apparatus $(T, N, B, \kappa, \tau)$. The curve $\alpha$ is a general helix, if and only if the harmonic curvature function of the curve $\alpha$ is a constant function. 
Proof. It is obvious using Definition 3 and Theorem 1.

Theorem 3 ([19]). Let $\alpha: I \subset \mathbb{R} \rightarrow G$ be a unit speed curve with the Frenet apparatus $(T, N, B, \kappa, \tau)$. Then $\alpha$ is a slant helix if and only if

$$
\sigma_{N}=\frac{\kappa\left(1+H^{2}\right)^{\frac{3}{2}}}{H^{1}}=\tan \theta
$$

is a constant where $H$ is a harmonic curvature function of the curve $\alpha$ and $\theta \neq \frac{\pi}{2}$ is a constant.

Theorem 4 ([12]). Let $\alpha: I \subset \mathbb{R} \rightarrow G$ be a parametrized curve with arc length parameter $s$ and the Frenet apparatus $(T, N, B, \kappa, \tau)$. Then, $\alpha$ is Mannheim curve if and only if

$$
\lambda \kappa\left(1+H^{2}\right)=1, \text { for all } s \in I
$$

where $\lambda$ is constant and $H$ is the harmonic curvature function of the curve $\alpha$.

Theorem 5. Let $\alpha: I \subset \mathbb{R} \rightarrow G$ be a parametrized curve with arc length parameter $s$. Then $\beta$ is the Mannheim partner curve of $\alpha$ if and only if the curvature $\kappa_{\beta}$ and the torsion $\tau_{\beta}$ of $\beta$ satisfy the following equation

$$
\frac{d \kappa_{\beta} H_{\beta}}{d \bar{s}}=\frac{\kappa_{\beta}}{\mu}\left(1+\mu^{2} \kappa_{\beta}^{2} H_{\beta}^{2}\right)
$$

where $\mu$ is constant and $H_{\beta}$ is the harmonic curvature function of the curve $\beta$.

\section{BERTRAND CURVES IN A THREE DIMENSIONAL LIE GROUP}

In this section, we define Bertrand curves and their characterizations are given in a three dimensional Lie group $G$ with a bi-invariant metric $\langle$,$\rangle . Also we give some$ characterizations of Bertrand curves using the special cases of $G$.

Definition 4. A curve $\alpha$ in 3-dimensional Lie group $G$ is a Bertrand curve if there exists a special curve $\beta$ in 3-dimensional Lie group $G$ such that principal normal vector field of $\alpha$ is linearly dependent principal normal vector field of $\beta$ at corresponding point under $\psi$ which is bijection from $\alpha$ to $\beta$. In this case $\beta$ is called the Bertrand mate curve of $\alpha$ and $(\alpha, \beta)$ is called Bertrand curve couple.

The curve $\alpha: I \subset \mathbb{R} \rightarrow G$ in 3-dimensional Lie group $G$ is parametrized by the arc-length parameter $s$ and from Definition 4 Bertrand mate curve of $\alpha$ is given $\beta$ : $\bar{I} \subset \mathbb{R} \rightarrow G$ in 3-dimensional Lie group $G$ with the help of Figure 1 such that

$$
\beta(s)=\alpha(s)+\lambda(s) N(s), s \in I
$$

where $\lambda$ is a smooth function on $I$ and $N$ is the principal normal vector field of $\alpha$. We should remark that the parameter $s$ generally is not an arc-length parameter of $\beta$. 


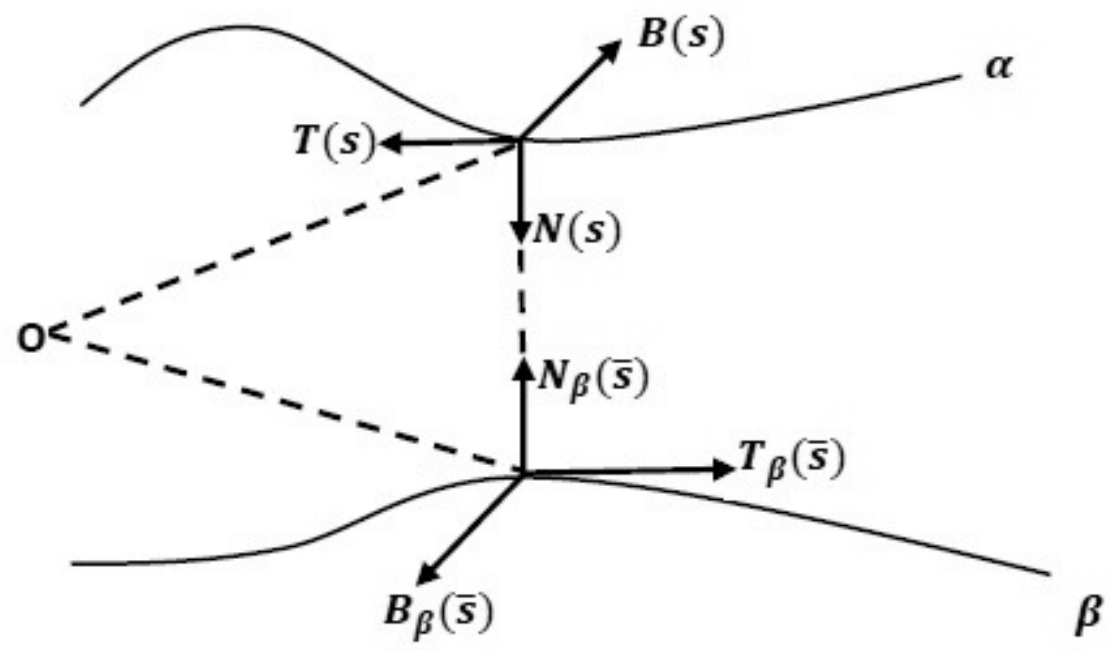

FiguRE 1. Bertrand Partner Curves

So, we define the arc-length parameter of the curve $\beta$ by

$$
\bar{s}=\psi(s)=\int_{0}^{s}\left\|\frac{d \beta(s)}{d s}\right\| d s
$$

where $\psi: I \longrightarrow \bar{I}$ is a smooth function and holds the following equality

$$
\psi^{\prime}(s)=\kappa H \sqrt{\lambda^{2}+\mu^{2}}
$$

for $s \in I$.

Proposition 2 ([19]). Let $\alpha: I \subset \mathbb{R} \rightarrow G$ be an arc length parametrized curve with the Frenet apparatus $\{T, N, B\}$. Then the following equalities

$$
\begin{aligned}
& {[T, N]=\langle[T, N], B\rangle B=2 \tau_{G} B} \\
& {[T, B]=\langle[T, B], N\rangle N=-2 \tau_{G} N}
\end{aligned}
$$

hold.

Theorem 6. Let $\alpha: I \subset \mathbb{R} \rightarrow G$ and $\beta: \bar{I} \subset \mathbb{R} \rightarrow G$ be a Bertrand curve couple with arc-length parameter $s$ and $\bar{s}$, respectively. Then corresponding points are a fixed distance apart for all $s \in I$, that is,

$$
d(\alpha(s), \beta(s))=\text { constant, for all } s \in I
$$

Proof. From Definition 4, we can simply write

$$
\beta(s)=\alpha(s)+\lambda(s) N(s)
$$


Differentiating Eq. (3.2) with respect to $s$ and using Eq. (2.3), we get

$$
\begin{aligned}
\frac{d \beta(\bar{s})}{d \bar{s}} \psi^{\prime}(s) & =\frac{d \alpha(s)}{d s}+\lambda^{\prime}(s) N(s)+\lambda(s) \dot{N}(s) \\
& =(1-\lambda(s) \kappa(s)) T(s)+\lambda^{\prime}(s) N(s)+\lambda(s) \tau(s) B(s)-\frac{1}{2}[T, N]
\end{aligned}
$$

and with the help of Proposition 2, we obtain

$$
\frac{d \beta(\bar{s})}{d \bar{s}} \psi^{\prime}(s)=(1-\lambda(s) \kappa(s)) T(s)+\lambda^{\prime}(s) N(s)+\lambda(s)\left(\left(\tau-\tau_{G}\right)(s)\right) B(s)
$$

or

$$
T_{\beta}(\bar{s})=\frac{1}{\psi^{\prime}(s)}\left[(1-\lambda(s) \kappa(s)) T(s)+\lambda^{\prime}(s) N(s)+\lambda(s)\left(\left(\tau-\tau_{G}\right)(s)\right) B(s)\right] .
$$

And then, we know that $\left\{N_{\beta}((\bar{s})), N(s)\right\}$ is a linearly dependent set, so we have

$$
\left\langle T_{\beta}(\bar{s}), N_{\beta}(\bar{s})\right\rangle=\frac{1}{\psi^{\prime}(s)}\left[\begin{array}{c}
(1-\lambda(s) \kappa(s))\left\langle T(s), N_{\beta}(\bar{s})\right\rangle+\lambda^{\prime}(s) \\
+\lambda(s) \tau(s)\left\langle B(s), N_{\beta}(\bar{s})\right.
\end{array}\right)
$$

Since $\left\langle T_{\beta}(\bar{s}), N_{\beta}(\bar{s})\right\rangle=0$, we get $\lambda^{\prime}(s)=0$ from the last formula. That is, $\lambda(s)$ is a constant function on $I$. This completes the proof.

Theorem 7. If $\alpha: I \subset \mathbb{R} \rightarrow G$ is a parametrized Bertrand curve with arc length parameter $s$ and the Frenet apparatus $(T, N, B, \kappa, \tau)$, then $\alpha$ satisfy the following equality

$$
\lambda \kappa(s)+\mu \kappa(s) H(s)=1, \text { for all } s \in I
$$

where $\lambda, \mu$ are constants and $H$ is the harmonic curvature function of the curve $\alpha$.

Proof. Let $\alpha: I \subset \mathbb{R} \rightarrow G$ be a parametrized Bertrand curve with arc length parameter $s$ then we can write

$$
\beta(s)=\alpha(s)+\lambda N(s)
$$

Differentiating the above equality with respect to $s$ and by using the Frenet equations, we get

$$
\begin{aligned}
\frac{d \beta(\bar{s})}{d \bar{s}} \psi^{\prime}(s) & =\frac{d \alpha(s)}{d s}+\lambda(s) \dot{N} \\
& =(1-\lambda(s) \kappa(s)) T(s)+\lambda(s) \tau(s) B(s)-\frac{1}{2}[T, N]
\end{aligned}
$$

and with the help of Proposition 2, we obtain

$$
T_{\beta}(\bar{s})=\frac{(1-\lambda \kappa(s))}{\psi^{\prime}(s)} T(s)+\frac{\lambda\left(\left(\tau-\tau_{G}\right)(s)\right)}{\psi^{\prime}(s)} B(s) .
$$

As $\left\{N_{\beta}((\bar{s})), N(s)\right\}$ is a linearly dependent set, we can write

$$
T_{\beta}(\bar{s})=\cos \theta(s) T(s)+\sin \theta(s) B(s)
$$


where

$$
\begin{aligned}
& \cos \theta(s)=\frac{(1-\lambda \kappa(s))}{\psi^{\prime}(s)}, \\
& \sin \theta(s)=\frac{\lambda\left(\left(\tau-\tau_{G}\right)(s)\right)}{\psi^{\prime}(s)} .
\end{aligned}
$$

If we differentiate Eq. (3.4) and consider $\left\{N_{\beta}(\bar{s}), N(s)\right\}$ is a linearly dependent set we can easily see that $\theta$ is a constant function. So, we obtain

$$
\frac{\cos \theta}{\sin \theta}=\frac{1-\lambda \kappa(s)}{\lambda\left(\left(\tau-\tau_{G}\right)(s)\right)}
$$

or taking $c=\frac{\cos \theta}{\sin \theta}$, we get

$$
\lambda \kappa(s)+c \lambda\left(\left(\tau-\tau_{G}\right)(s)\right)=1 .
$$

Then denoting $\mu=c \lambda=$ constant and using Definition 3, we have

$$
\lambda \kappa(s)+\mu \kappa(s) H(s)=1, \text { for all } s \in I,
$$

which completes the proof.

Corollary 1. The measure of the angle between the tangent vector fields of the Bertrand curve couple $(\alpha, \beta)$ is constant.

Proof. It is obvious from the proof of the above Theorem.

Remark 1. It is unknown whether the reverse of the above Theorem holds. Because, for the proof of the reverse we must consider a special Frenet curve $\beta(s)=$ $\alpha(s)+\lambda N(s)$ in its proof. So, we give the following Theorem.

Theorem 8. Let $\alpha: I \subset \mathbb{R} \rightarrow G$ be a parametrized Bertrand curve whose curvature functions $\kappa$ and harmonic curvature function $H$ of the curve $\alpha$ satisfy $\lambda \kappa(s)+$ $\mu \kappa(s) H(s)=1$, for all $s \in I$. If the curve $\beta$ given by $\beta(s)=\alpha(s)+\lambda N(s)$ for all $s \in I$ is a special Frenet curve, then $(\alpha, \beta)$ is the Bertrand curve couple.

Proof. Let $\alpha: I \subset \mathbb{R} \rightarrow G$ be a parametrized Bertrand curve whose curvature function $\kappa$ and harmonic curvature function $H$ of the curve $\alpha$ satisfy $\lambda \kappa(s)+$ $\mu \kappa(s) H(s)=1$ for all $s \in I$. If the curve $\beta$ given by $\beta(s)=\alpha(s)+\lambda N(s)$ for all $s \in I$ is a special Frenet curve, then differentiating this equality with respect to $s$ and by using Eq. (3.1) with the equation $\lambda \kappa(s)+\mu \kappa(s) H(s)=1$, we have

$$
T_{\beta}(\bar{s})=\frac{\mu}{\sqrt{\lambda^{2}+\mu^{2}}} T(s)+\frac{\lambda}{\sqrt{\lambda^{2}+\mu^{2}}} B(s) .
$$

Then, if we differentiate the last equation with respect to $s$ and by using the Frenet formulas we obtain

$$
\kappa_{\beta}(\bar{s}) N_{\beta}(\bar{s}) \psi^{\prime}(s)=\frac{\kappa(s)}{\sqrt{\lambda^{2}+\mu^{2}}}(\mu-\lambda H(s)) N(s) .
$$


Thus, for each $s \in I$, the vector field $N_{\beta}(\bar{s})$ of $\beta$ is linearly dependent the vector field $N(s)$ of $\alpha$ at corresponding point under the bijection from $\alpha$ to $\beta$. This completes the proof.

Proposition 3. Let $\alpha: I \subset \mathbb{R} \rightarrow G$ be an arc-lenghted Bertrand curve with the Frenet vector fields $\{T, N, B\}$ and $\beta: \bar{I} \subset \mathbb{R} \rightarrow G$ be a Bertrand mate of $\alpha$ with the Frenet vector fields $\left\{T_{\beta}, N_{\beta}, B_{\beta}\right\}$. Then $\tau_{G_{\beta}}=\tau_{G}$ for the curves $\alpha$ and $\beta$ where $\tau_{G}=\frac{1}{2}\langle[T, N], B\rangle$ and $\tau_{G \beta}=\frac{1}{2}\left\langle\left[T_{\beta}, N_{\beta}\right], B_{\beta}\right\rangle$.

Proof. Let $\alpha: I \subset \mathbb{R} \rightarrow G$ be an arc-lenghted Bertrand curve with the Frenet vector fields $\{T, N, B\}$ and $\beta: \bar{I} \subset \mathbb{R} \rightarrow G$ be a Bertrand mate of $\alpha$ with with the Frenet vector fields $\left\{T_{\beta}, N_{\beta}, B_{\beta}\right\}$. From Eq. (3.5) and considering $N_{\beta}=\mp N$ we have

$$
B_{\beta}(\bar{s})=-\frac{\lambda}{\sqrt{\lambda^{2}+\mu^{2}}} T(s)+\frac{\mu}{\sqrt{\lambda^{2}+\mu^{2}}} B(s) .
$$

Since $\tau_{G \beta}=\frac{1}{2}\left\langle\left[T_{\beta}, N_{\beta}\right], B_{\beta}\right\rangle$, using the equalities of the Frenet vector fields $T_{\beta}, N_{\beta}$ and $B_{\beta}$ we obtain $\tau_{G \beta}=\tau_{G}$, which completes the proof.

Theorem 9. Let $\alpha: I \subset \mathbb{R} \rightarrow G$ be a parametrized Bertrand curve with curvature functions $\kappa, \tau$ and $\beta: \bar{I} \subset \mathbb{R} \rightarrow G$ be a Bertrand mate of $\alpha$ with curvatures functions $\kappa_{\beta}$, $\tau_{\beta}$. Then the relations between these curvature functions are

$$
\begin{aligned}
\kappa_{\beta}(\bar{s}) & =\frac{\mu \kappa(s)-\lambda \kappa(s) H(s)}{\left(\lambda^{2}+\mu^{2}\right) H(s)}, \\
\tau_{\beta}(\bar{s}) & =\frac{\lambda \kappa(s)+\mu \kappa(s) H(s)}{\left(\lambda^{2}+\mu^{2}\right) H(s)}+\tau_{G}
\end{aligned}
$$

Proof. If we take the norm of Eq. (3.6) and use Eq. (3.1), we get Eq. (3.8). Then differentiating Eq. (3.7) and using the Frenet formulas, we have

$$
\begin{aligned}
\dot{B}_{\beta}(\bar{s}) \psi^{\prime}(s) & =-\frac{\lambda}{\sqrt{\lambda^{2}+\mu^{2}}} \dot{T}(s)+\frac{\mu}{\sqrt{\lambda^{2}+\mu^{2}}} \dot{B}(s), \\
& =-\frac{\lambda}{\sqrt{\lambda^{2}+\mu^{2}}} \kappa(s) N(s)+\frac{\mu}{\sqrt{\lambda^{2}+\mu^{2}}}\left(-\tau(s) N(s)-\frac{1}{2}[T, B]\right)
\end{aligned}
$$

In the above equality, using Eq. (3.1) and Proposition 2, we get

$$
\left(\tau_{\beta}-\tau_{G \beta}\right) N_{\beta}(\bar{s})=\frac{1}{\kappa H\left(\lambda^{2}+\mu^{2}\right)}(\lambda \kappa+\mu \kappa H) N(s) .
$$

If we take the norm of the last equation and use Proposition 3, we get Eq. (3.9), which completes the proof.

Theorem 10. Let $\alpha: I \subset \mathbb{R} \rightarrow G$ be a parametrized curve with Frenet apparatus $\{T, N, B, \kappa, \tau\}$ and $\beta: \bar{I} \subset \mathbb{R} \rightarrow G$ be a curve with Frenet apparatus 
$\left\{T_{\beta}, N_{\beta}, B_{\beta}, \kappa_{\beta}, \tau_{\beta}\right\}$. If $(\alpha, \beta)$ is a Bertrand curve couple then $\kappa_{\beta} H H_{\beta}$ is a constant function.

Proof. We assume that $(\alpha, \beta)$ is a Bertrand curve couple. Then we can write

$$
\alpha(s)=\beta(s)-\lambda(s) N_{\beta}(\bar{s}) .
$$

If we use the similar method as in the proof of Theorem 7 and consider Eq. (3.10), then we can easily see that $\kappa_{\beta} H H_{\beta}$ is a constant function.

Theorem 11. Let $\alpha: I \subset \mathbb{R} \rightarrow G$ be a parametrized Bertrand curve with Frenet apparatus $\{T, N, B, \kappa, \tau\}$ and $\beta: \bar{I} \subset \mathbb{R} \rightarrow G$ be a Bertrand mate of the curve $\alpha$ with Frenet apparatus $\left\{T_{\beta}, N_{\beta}, B_{\beta}, \kappa_{\beta}, \tau_{\beta}\right\}$. Then $\alpha$ is a slant helix if and only if $\beta$ is a slant helix.

Proof. Let $\sigma_{N}$ and $\sigma_{N \beta}$ be the geodesic curvatures of the principal normal curves of $\alpha$ and $\beta$, respectively. Then using Theorem 9 we can easily see that

$$
\sigma_{N \beta}=-\frac{\kappa\left(1+H^{2}\right)^{\frac{3}{2}}}{H^{1}}=-\sigma_{N} \text {. }
$$

So, with the help of Theorem 3 we complete the proof.

Theorem 12. Let $\alpha: I \subset \mathbb{R} \rightarrow G$ be a parametrized Bertrand curve with curvature functios $\kappa, \tau$ and $\beta: \bar{I} \subset \mathbb{R} \rightarrow G$ be a Bertrand mate of the curve $\alpha$ with curvature functions $\kappa_{\beta}, \tau_{\beta}$. Then $\alpha$ is a general helix if and only if $\beta$ is a general helix.

Proof. Let $\alpha$ be a helix. From Theorem 1, we have that $H$ is a constant function. Then using Theorem 9, we get

$$
\frac{\tau_{\beta}-\tau_{G \beta}}{\kappa_{\beta}}=\frac{\lambda+\mu H}{\mu-\lambda H} .
$$

Since $H$ is a constant function, Eq. (3.11) is constant. So, $\beta$ is a general helix.

Conversely, assume that $\beta$ be a general helix. So, $\frac{\tau_{\beta}-\tau_{G \beta}}{\kappa_{\beta}}=$ constant. From Eq. (3.11) $c=\frac{\lambda+\mu H}{\mu-\lambda H}=$ constant and then $H=\frac{c \mu-\lambda}{\mu+\lambda c}=$ constant. Consequently $\alpha$ is a general helix and this completes the proof.

\section{ACKNOWLEDGMENTS}

The authors would like to thank the anonymous referees for their helpful suggestions and comments which improved significantly the presentation of the paper.

\section{REFERENCES}

[1] H. Balgetir, M. Bektaş, and M. Ergüt, "Bertrand curves for non null curves in 3-dimensional Lorentzian space.” Hadronic J., vol. 27, no. 2, pp. 229-236, 2004.

[2] H. Balgetir, M. Bektaş, and J. Inoguchi, "Null Bertrand curves in Minkowski 3-space and their characterizations." Note Mat., vol. 23, no. 1, pp. 7-13, 2004. 
[3] J. M. Bertrand, "Mémoire sur la théorie des courbes á double courbure." Comptes Rendus, vol. 36, 1850.

[4] C. Bioche, "Sur les courbes de M. Bertrand." Bull. Soc. Math. France, vol. 17, pp. 109-112, 1889.

[5] J. F. Burke, "Bertrand Curves Associated with a Pair of Curves." Mathematics Magazine, vol. 34, no. 1, pp. 60-62, 1960, doi: 10.2307/2687860.

[6] Ç. Camcı, K. İlarslan, L. Kula, and H. H. Hacısalihoğlu, "Harmonic curvatures and generalized helices in $E^{n}$." Chaos, Solitons \& Fractals, vol. 40, no. 5, pp. 2590-2596, 2007, doi: 10.1016/j.chaos.2007.11.001.

[7] Ü. Çiftçi, “A generalization of Lancert's theorem,” J. Geom. Phys., vol. 59, no. 12, pp. 1597-1603, 2009, doi: 10.1016/j.geomphys.2009.07.016.

[8] A. C. Çöken and Ü. Çiftçi, “A note on the geometry of Lie groups," Nonlinear Analysis TMA, vol. 68, no. 7, pp. 2013-2016, 2008, doi: 10.1016/j.na.2007.01.028.

[9] P. Crouch and F. Silva Leite, "The dynamic interpolation problem: on Riemannian manifolds, Lie groups and symmetric spaces." J. Dyn. Control Syst., vol. 1, no. 2, pp. 177-202, 1995, doi: $10.1007 / \mathrm{bf02254638.}$

[10] N. Ekmekci and K. İlarslan, "On Bertrand curves and their characterization.” Differ. Geom. Dyn. Syst., vol. 3, no. 2, pp. 17-24, 2001.

[11] İ. Gök, Ç. Camc1, and H. H. Hacısalihoğlu, " $V_{n}$-slant helices in Euclidean n-space $E^{n}$," Math Commun., vol. 14, no. 2, pp. 317-329, 2009.

[12] İ. Gök, O. Z. Okuyucu, N. Ekmekci, and Y. Yayl1, "On Mannheim partner curves in three dimensional Lie groups," Miskolc Mathematical Notes, vol. 15, no. 2, pp. 467-479, 2014.

[13] S. Izumiya and N. Tkeuchi, "New special curves and developable surfaces." Turk. J. Math., vol. 28, pp. 153-163, 2004.

[14] D. H. Jin, "Null Bertrand curves in a Lorentz manifold." J. Korea Soc. Math. Educ. Ser. B: Pure Appl. Math., vol. 15, no. 3, pp. 209-215, 2008.

[15] W. Kuhnel, Differential geometry: curves-surfaces-manifolds, Braunschweig, Wiesbaden, 1999.

[16] M. Külahcı, M. Bektaş, and M. Ergüt, "On Harmonic curvatures of Frenet curve in Lorentzian space." Chaos, Solitons \& Fractals, vol. 41, no. 4, pp. 1668-1675, 2009, doi: 10.1016/j.chaos.2008.07.013.

[17] M. Külahcı and M. Ergüt, "Bertrand curves of AW(k)-type in Lorentzian space." Nonlinear Analysis TMA, vol. 70, no. 4, pp. 1735-1734, 2009.

[18] H. Matsuda and S. Yorozu, "Notes on Bertrand curves." Yokohama Math. J., vol. 50, pp. 41-58, 2003.

[19] O. Z. Okuyucu, İ. Gök, Y. Yaylı, and N. Ekmekci, "Slant Helices in three Dimensional Lie Groups," Appl. Math. Comput., vol. 221, pp. 672-683, 2013, doi: 10.1016/j.amc.2013.07.008.

[20] E. Özdamar and H. H. Hacisalihoğlu, "A characterization of inclined curves in Euclidean n-space," Commun. Fac. Sci. Univ. Ank. Ser. A1:Math. Stat., vol. 24, pp. 15-23, 1975, doi: 10.1501/Commua1_0000000261.

[21] L. R. Pears, "Bertrand curves in Riemannian space.” J. London Math. Soc., vol. s1-10, no. 2, pp. 180-183, 1935, doi: 10.1112/jlms/s1-10.2.180.

[22] J. K. Whittemore, "Bertrand curves and helices," Duke Math. J., vol. 6, no. 1, pp. 235-245, 1940, doi: 10.1215/S0012-7094-40-00618-4.

[23] M. Yıldırım Yılmaz and M. Bektaş, "General properties of Bertrand curves in RiemannOtsuki space," Nonlinear Analysis TMA, vol. 69, no. 10, pp. 3225-3231, 2008, doi: 10.1016/j.na.2007.10.003. 
Authors' addresses

\section{O. Zeki Okuyucu}

Bilecik Şeyh Edebali University, Faculty of Science and Arts, Department of Mathematics, 11210, Bilecik, Turkey

E-mail address: osman.okuyucu@bilecik.edu.tr

İsmail Gök

Ankara University, Faculty of Science, Department of Mathematics, 06100, Ankara, Turkey

E-mail address: igokescience.ankara.edu.tr

\section{Yusuf Yaylı}

Ankara University, Faculty of Science, Department of Mathematics, 06100, Ankara, Turkey

E-mail address: yayliescience. ankara.edu.tr

Nejat Ekmekci

Ankara University, Faculty of Science, Department of Mathematics, 06100, Ankara, Turkey

E-mail address: ekmekci@science.ankara.edu.tr 